\title{
Abdomen agudo quirúrgico. Un reto diagnóstico
}

\author{
Acute surgical abdomen: a diagnostic challenge
}

Salvador Eloy García-Valenzuela, * Pablo Valente Bonilla-Catalán,* Benjamín Quintero-García, ${ }^{* *}$ Francisco Samuel Trujillo-Bracamontes, ${ }^{* *}$ José del Carmen Ríos-Beltrán, ${ }^{* *}$ Jaime Alberto Sánchez-Cuén, ${ }^{* * *}$ Denisse Valdez-Avilés****

\section{Palabras clave: \\ Dolor abdominal, abdomen agudo, servicios de urgencias, diagnóstico, evaluación, educación médica. \\ Key words: Abdominal pain, acute abdomen, emergency services, diagnosis, evaluation, medical education.}

* Médico residente. Servicio de Cirugía General.

** Médico adscrito. Servicio de Cirugía General.

*** Médico

adscrito. Servicio de

Gastroenterología

y Departamento de

Postgrado de la Facultad de Medicina de la

Universidad Autónoma de Sinaloa.

***** Médica General.

Servicio de Urgencias.

Hospital Regional "Dr. Manuel Cárdenas de la Vega", Instituto de Seguridad y Servicios Sociales de los Trabajadores del Estado. Culiacán, Sinaloa, México.

\section{RESUMEN}

Introducción: El dolor abdominal es un síntoma muy frecuente originado por un amplio espectro de enfermedades y trastornos que son, en su mayoría, de origen benigno; sin embargo, en algunas situaciones, se debe a un proceso grave o enfermedad que pone en peligro la vida. Objetivo: Determinar cuántas consultas se requieren para el diagnóstico de abdomen agudo quirúrgico. Material y métodos: Se realizó una cohorte en pacientes operados por diagnóstico de abdomen agudo quirúrgico en el Hospital Regional de Culiacán del ISSSTE, en el periodo de septiembre de 2015 a enero de 2016. Resultados: La muestra consistió en un total de 56 pacientes operados por abdomen agudo; las causas más frecuentes fueron apendicitis aguda (62.5\%) y patología biliar (23.3\%). En el 41.1\% (IC 95\%: [28.1, $55.0])$ de los pacientes hubo cambio en el diagnóstico inicial. En total, fueron 69 consultas de valoración entre los 56 pacientes operados, quienes tuvieron que acudir un promedio de $1.3 \pm .5$ veces a consulta. Conclusiones: Se requieren hasta dos consultas para el diagnóstico de abdomen agudo quirúrgico; al incrementar el número de consultas aumenta hasta 12 veces la probabilidad de un diagnóstico erróneo.

\section{ABSTRACT}

Introduction: Abdominal pain is a very common symptom, caused by a broad spectrum of diseases and disorders, most of which have a benign origin; however, in some situations, the cause is a serious process or life-threatening disease. Objective: To determine how many consultations are required for the diagnosis of acute surgical abdomen. Material and methods: We conducted a cohort of patients operated on with a diagnosis of acute surgical abdomen at Culiacan's Regional Hospital, ISSSTE, in the period from September 2015 to January 2016. Results: The sample consisted of a total of 56 patients operated on for acute abdomen; the most frequent causes were acute appendicitis (62.5\%) and biliary pathology (23.3\%). In $41.1 \%$ (IC 95\%: [28.1, 55.0]) of the patients, there was a change in the initial diagnosis. There were 69 valuation consultations among the 56 operated patients, who had to undergo an average of $1.3 \pm .5$ consultations. Conclusions: Up to two consultations were required for the diagnosis of acute surgical abdomen; when the number of consultations is higher, the probability of an erroneous diagnosis increases up to 12 times.

\section{INTRODUCCIÓN}

$\mathrm{E}$ I dolor abdominal es un síntoma muy frecuente originado por un amplio espectro de enfermedades y trastornos que son, en la mayoría de los casos, de origen benigno; sin embargo, en algunas situaciones, se debe a un proceso grave o enfermedad que pone en peligro la vida. ${ }^{1-25}$ En Estados Unidos de América (EUA), se estima que más de siete millones de pacientes acuden al servicio de urgencias por dolor abdominal; esto representa el $6.5 \%$ de todas las consultas que se tienen en el servicio de urgencias. $2,7,9-11,15,21,23,25$ En México, comprende aproximadamente el $30 \%$ del total de las consultas de urgencias y constituye entre el 13 y $40 \%$ de todas las emergencias posiblemente quirúrgicas, cuando se trata de dolor abdominal agudo no específico. ${ }^{1,12}$

Se estima que el $50 \%$ de los casos de dolor abdominal agudo requieren hospitalización, y entre el 30 y $40 \%$, cirugía. ${ }^{4-6} \mathrm{Al}$ ingreso hospitalario, cerca de $40 \%$ se diagnostican de forma errónea, por lo que su mortalidad global es del $10 \%$ y se eleva al $20 \%$ si el paciente requiere cirugía de urgencia., 3,5,

El abdomen agudo es un reto para el cirujano general, sobre todo en casos con duda de diagnóstico; es una importante causa de 
morbimortalidad. La decisión de recurrir a cirugía o brindar tratamiento médico (el cual, por lo general, es difícil) requiere la asimilación completa de la información que proporcionan el paciente o sus familiares. ${ }^{1-25}$

El síndrome de abdomen agudo se define como el conjunto de signos y síntomas que tiene como base el dolor abdominal intenso de inicio brusco o insidioso, de rápida evolución (menor de 24 o 48 horas) y que puede ser de origen abdominal o extraabdominal. El dolor suele acompañarse de uno o más signos de irritación peritoneal; entre los más frecuentes se encuentran: rigidez de la musculatura abdominal, incremento de la sensibilidad abdominal con o sin signo de rebote positivo, defensa o resistencia involuntaria. $1,7,8$

El síndrome de abdomen agudo se clasifica en no quirúrgico y quirúrgico; este último se presenta con una frecuencia que va del 10 al $25 \%$. $^{3,5,6}$ Las causas quirúrgicas pueden dividirse en cinco grupos: inflamación/infección, perforación, obstrucción, hemorragia e isquemia, y no son mutuamente excluyentes. El diagnóstico varía dependiendo de la edad y el sexo del paciente. La apendicitis es más frecuente en los jóvenes, mientras que los trastornos biliares, la obstrucción intestinal, la isquemia, el infarto intestinal y la diverticulitis son más frecuentes en los pacientes de edad avanzada. 7,14,15,22

Debido al posible carácter quirúrgico del abdomen agudo, es necesario realizar una evaluación inmediata y completa; lo más importante es la historia clínica exhaustiva y la exploración física minuciosa, aunque en ocasiones, los hallazgos clínicos son insuficientes para llegar a un diagnóstico, por los que se necesita de exámenes complementarios, como pruebas de laboratorio, ultrasonido o tomografía de abdomen. ${ }^{1,2,7,9-11}$

Por tratarse de una de las patologías más frecuentes en los servicios de urgencias y debido a la complejidad para llegar al diagnóstico etiológico del abdomen agudo, decidimos realizar este trabajo, para determinar las causas etiológicas más frecuentes de abdomen agudo quirúrgico y el número de consultas que recibieron los pacientes en el servicio de urgencias por los médicos de primer contacto.

\section{MATERIAL Y MÉTODOS}

Se trata de un estudio de cohorte, la población fueron pacientes que acudieron al servicio de urgencias con dolor abdominal y que fueron hospitalizados para su estudio y tratamiento en el periodo de septiembre de 2015 a enero de 2016.

Los criterios de inclusión fueron pacientes mayores de 18 años, derechohabientes del servicio de salud, de ambos géneros y que estuvieran internados en el servicio de urgencias con diagnóstico de dolor abdominal. Se excluyeron las pacientes obstétricas y ginecológicas, aquéllos con abdomen agudo postquirúrgico, quienes tenían enfermedades psiquiátricas o estaban en estado de coma; se eliminaron del estudio todos aquellos pacientes con falta de seguimiento.

Los pacientes fueron valorados por médicos residentes de $1^{\circ}, 2^{\circ}, 3^{\circ}$ y $4^{\circ}$ año de la Especialidad de Cirugía General, supervisados por el cirujano general encargado de las urgencias quirúrgicas, para determinar si el dolor abdominal contaba con criterios de abdomen agudo quirúrgico.

Los datos categóricos se resumen con frecuencias y porcentajes, y los numéricos, con medias y desviación estándar. Para comparar los grupos en relación con variables categóricas, se utilizó la prueba $\chi^{2}$ y el índice de kappa para medir concordancia. Se estimó la razón de momios con intervalos de confianza del 95\% para relacionar el número de consultas y el cambio en el diagnóstico. Para comparar medias, se utilizó la prueba t-Student. Los datos fueron analizados en SPSS v11. Un valor de probabilidad menor a .05 se consideró estadísticamente significativo.

\section{RESULTADOS}

La muestra consistió en un total de 56 pacientes, 24 de género femenino (42.9\%) y 32 (57.1\%) masculino, con edad entre los 18 y los 89 años, con un promedio de $49.1 \pm 20.3$ años. En el cuadro I se muestra la frecuencia y porcentaje de los signos y síntomas más comunes que se encontraron en la valoración por cirugía general y que determinaron si se trataba de abdomen agudo quirúrgico. 
Las causas más frecuentes de abdomen agudo quirúrgico fueron apendicitis aguda, en 35 pacientes (62.5\%), y patología biliar, en 13 (23.3\%) (Figura 1). La etiología se reporta en el cuadro II.

En el cuadro III se muestran las causas de morbilidad que se presentaron en los pacientes intervenidos quirúrgicamente.

\begin{tabular}{cccc}
\multicolumn{2}{c}{$\begin{array}{c}\text { Cuadro I. Frecuencia de los signos y } \\
\text { síntomas más comunes en la valoración médica. }\end{array}$} \\
\hline \multirow{2}{*}{ Tipo } & Estudios & Frecuencia & $\%$ \\
\hline \multirow{2}{*}{ Signos } & Fiebre & 19 & 33.9 \\
& Deshidratación & 23 & 41.1 \\
Palidez & 20 & 35.7 \\
Rebote & 41 & 73.2 \\
Síntomas & Resistencia muscular & 33 & 58.9 \\
& Rigidez muscular & 25 & 44.6 \\
& Murphy & 13 & 23.2 \\
& McBurney & 23 & 41.1 \\
& Dolor abdominal & 52 & 92.9 \\
Náuseas & 29 & 51.8 \\
& Vómitos & 19 & 33.9 \\
& Estreñimiento & 13 & 23.6
\end{tabular}

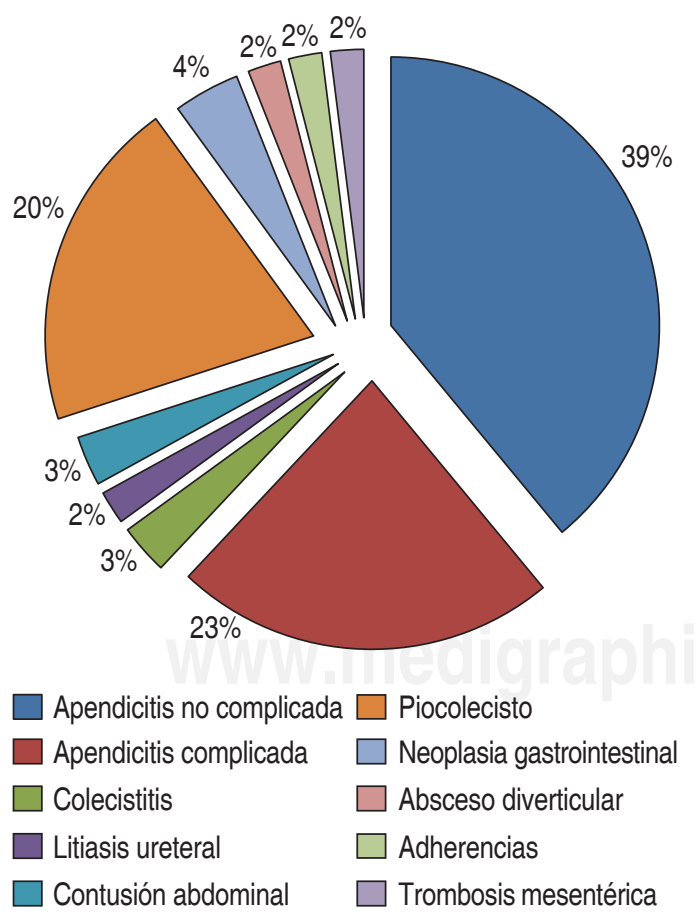

Figura 1. Frecuencia de abdomen agudo quirúrgico.
No se observó concordancia entre el diagnóstico inicial y final, kappa $=.428, \mathrm{p}=.000$. La mayoría de los diagnósticos iniciales fueron de apendicitis aguda, 22 (39.3\%), seguida de piocolecisto, con nueve (16.1\%) de casos. De los 35 casos con diagnóstico final de apendicitis, 20 tuvieron diagnóstico inicial de apendicitis aguda (57.1\%) y otros ocho, diferentes diagnósticos iniciales.

De los 56 pacientes, en 23 (41.1\%), IC 95\%: (28.1, 55.0), se observó cambio en el diagnóstico; es decir, diagnóstico inicial erróneo.

Del total de los 23 casos de cambio en el diagnóstico, los más frecuentes fueron cuatro casos de gastroenteritis (que en realidad fueron apendicitis), tres diagnósticos de intestino irritable (fueron, al final, apendicitis) y tres de litiasis ureteral (también con diagnóstico final de apendicitis).

El cuadro IV describe el total de consultas que tuvo el médico de primer contacto en el

\begin{tabular}{ccr} 
Cuadro II. Etiología del abdomen agudo. \\
& Frecuencia & $\%$ \\
\hline Infección & 32 & 57.1 \\
Obstrucción & 2 & 3.6 \\
Isquémica & 1 & 1.8 \\
Mixta & 19 & 33.9 \\
Total & 54 & 96.4 \\
NR & 2 & 3.6 \\
Total & 56 & 100.0
\end{tabular}

\begin{tabular}{|c|c|c|}
\hline \multicolumn{3}{|c|}{ Cuadro III. Morbilidad. } \\
\hline & Frecuencia & $\%$ \\
\hline \multirow{7}{*}{ 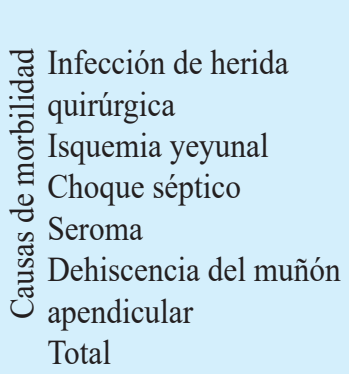 } & 5 & 8.9 \\
\hline & 1 & 1.8 \\
\hline & 1 & 1.8 \\
\hline & 1 & 1.8 \\
\hline & 1 & 1.8 \\
\hline & 1 & 1.8 \\
\hline & 56 & 100 \\
\hline
\end{tabular}


Cuadro IV. Número de consultas promedio realizadas por paciente por etiología.

\begin{tabular}{lrc} 
Etiología & Suma & Media \\
\hline Infección & 39 & $1.2 \pm 0.4$ \\
Obstrucción & 2 & $1.0 \pm 0.0$ \\
Isquémica & 1 & $1.0 \pm 0.0$ \\
Mixta & 27 & $1.4 \pm 0.7$ \\
Total & 69 & $1.3 \pm 0.5$
\end{tabular}

servicio de urgencias y el promedio de consultas por paciente.

A medida que se incrementó el número de consultas al paciente, también aumentó el riesgo de cambio en el diagnóstico en más de 12 veces ( $R M=12.2$, IC 95\%: 2.4, 61.7).

\section{DISCUSIÓN}

En nuestro análisis, obtuvimos que el abdomen agudo quirúrgico es más frecuente en el hombre (57.1\%) que en la mujer (42.9\%); esto coincide con otros reportes, como el de Montalva y sus colaboradores, ${ }^{2}$ donde el sexo masculino fue el más frecuente, con el $53.9 \%$, y el de Casado y su grupo, ${ }^{3}$ con $58.24 \%$; sin embargo, difiere de Bejarano, ${ }^{1}$ Pérez y sus respectivos equipos, ${ }^{7}$ ya que ellos reportaron una frecuencia mayor en el sexo femenino para el abdomen agudo quirúrgico. Respecto a la edad, el rango fue de 18 a 89 años, esto por haber escogido la población adulta para el estudio; se obtuvo una media de 49 años para ambos sexos; este parámetro fue variable en las diferentes series reportadas. ${ }^{1-3,7}$

Los síntomas más frecuentes que presentaron los pacientes fueron dolor abdominal (92.9\%), náuseas $(51.8 \%)$, vómitos $(33.9 \%)$ y estreñimiento (23.6\%). Bejarano y sus colegas ${ }^{1}$ reportaron en su trabajo que los pacientes sometidos a cirugía por abdomen agudo quirúrgico presentaron como síntomas asociados más frecuentes el vómito $(50 \%)$, distensión (26.5\%), diarrea (17.6\%) y náuseas (14.7\%). Los signos más frecuentes fueron signo de rebote $(73.2 \%)$, resistencia muscular $(58.9 \%)$, rigidez muscular (44.6\%) y deshidratación (41.1\%). Bejarano y sus colaboradores ${ }^{1}$ describieron que en su estudio de los pacientes operados, los signos más frecuentes fueron defensa muscular voluntaria (11.8\%), signo de Murphy (14.7\%) y signo de Blumberg (8.8\%). El estudio diagnóstico imagenológico que más se realizó fue el US (57.1\%), seguido de las radiografías simples de abdomen (50\%); Ilama la atención que la TAC de abdomen se llevó a cabo sólo en el $8.9 \%$ de los casos. Montalva y su grupo ${ }^{2}$ reportaron que el estudio que más se realizó fue la TAC de abdomen y pelvis con contraste, seguida de la radiografía de abdomen simple de pie. En nuestro trabajo, se prefirió como paraclínico de imagen el US por ser la patología biliar la segunda en frecuencia, para diagnóstico diferencial de padecimiento ginecológico y en los casos de duda diagnóstica.

La principal causa de abdomen agudo quirúrgico fue la apendicitis aguda (62.5\%), de las cuales el $23.3 \%$ fueron complicadas. El segundo lugar lo ocupó la patología biliar (23.2\%); de éstas, el piocolecisto fue el más frecuente (19.6\%). En el estudio de Montalva y sus colegas, ${ }^{4}$ las principales causas de abdomen agudo fueron apendicitis aguda, úlcera gástrica perforada y colecistitis aguda. Bejarano y su equipo ${ }^{1}$ reportaron que en pacientes intervenidos quirúrgicamente, la causa principal fue la enfermedad biliar (colelitiasis y colecistitis), apendicitis aguda, hernias de la pared abdominal y obstrucción intestinal. En el estudio de Casado y sus colaboradores, ${ }^{3}$ las enfermedades causantes de abdomen agudo fueron apendicitis aguda, colecistitis aguda y obstrucción intestinal. En los pacientes geriátricos del estudio de Pérez y su grupo, ${ }^{7}$ las causas más frecuentes de intervención quirúrgica fueron enfermedades biliares (colecistitis aguda y piocolecisto), obstrucción intestinal por adherencias, hernias de pared abdominal, diverticulitis, trombosis mesentérica, tumores gastrointestinales y apendicitis del anciano. Las enfermedades causantes de abdomen agudo quirúrgico son muy similares en todos los trabajos, las variaciones en orden de frecuencia dependen del tipo de población predominante en cada región de estudio. En cuanto a la etiología del abdomen agudo quirúrgico, el origen más frecuente fue infeccioso (57.1\%), seguido del mixto (33.9\%). No encontramos en nuestra revisión algún artículo para comparar la etiología del abdomen 
agudo quirúrgico, pero creemos que por las similitudes de los diagnósticos postoperatorios, la frecuencia no debe ser muy variable.

De los 56 pacientes que fueron sometidos a cirugía, sólo en cinco (8.9\%) se presentaron complicaciones como infección de herida quirúrgica $(1.8 \%)$, seroma $(1.8 \%)$, dehiscencia del muñón apendicular (1.8\%), isquemia del segmento de intestino delgado (1.8\%) y choque séptico (1.8\%). En el reporte de Casado y sus colegas, ${ }^{5}$ las complicaciones más frecuentes fueron los trastornos hidroelectrolíticos y ácido/ base (28.5\%) y la infección del sitio quirúrgico (20\%). A pesar de que en nuestro estudio un gran número de cirugías fueron contaminadas, las complicaciones postquirúrgicas estuvieron con índices por debajo de lo descrito en la literatura y en artículos de reciente publicación.

Se analizó la concordancia entre el diagnóstico inicial en la consulta de urgencias y el diagnóstico final postoperatorio, y se encontró que en el $41.1 \%$ (IC 95\%: [28.1, 55.0]) de los pacientes hubo cambio en el diagnóstico inicial. Por ejemplo, de los 35 con diagnóstico postoperatorio de apendicitis, sólo en 20 (57.1\%) se sospechó de esta patología desde la consulta inicial; el resto de los operados por apendicitis tuvieron ocho diagnósticos iniciales diferentes, siendo los más frecuentes gastroenteritis, síndrome de intestino irritable y litiasis ureteral. En el estudio de Toorenvliet y su equipo, ${ }^{6}$ de 500 pacientes valorados por dolor abdominal, en el $30 \%$ de los casos el diagnóstico final fue diferente del diagnóstico de la evaluación inicial. El $17 \%$ tuvo un cambio en el manejo después de la reevaluación y el $4 \%$ fueron ingresados para cirugía.

Se realizaron en total 69 consultas de valoración en urgencias entre los 56 pacientes operados por abdomen agudo quirúrgico; se encontró que los pacientes tuvieron que acudir un promedio de $1.3 \pm .5$ veces a consulta en urgencias para ser diagnosticados con abdomen agudo quirúrgico, ser valorados y recibir el tratamiento adecuado por el cirujano. Además, se calculó que se aumenta 12 veces el riesgo de cambio en el diagnóstico inicial al incrementar el número de consultas para diagnosticar un abdomen agudo quirúrgico. Aunque no se halló algún artículo que trate sobre este tema en específico para compararlo, Brekke y sus colaboradores ${ }^{5}$ realizaron un estudio con médicos generales de primer contacto en el servicio de urgencias donde se observó que éstos se enfrentan a un gran reto por satisfacer un amplio y heterogéneo espectro de quejas cuando se trata de pacientes con dolor abdominal agudo, en los cuales la decisión diagnóstica y de tratamiento se debe hacer con herramientas simples; se reveló que el manejo fue muy variado y se ajustaba al problema específico planteado, y que cualquier presión sobre los médicos generales para ser más selectivos en las admisiones hospitalarias aumenta de manera inevitable el riesgo de retraso en el tratamiento de los pacientes gravemente enfermos.

Teniendo presente que el dolor abdominal es una de las causas más frecuentes de consulta en el servicio de urgencias, con nuestro estudio concluimos que para el diagnóstico oportuno del abdomen agudo quirúrgico, se requiere de un adecuado conocimiento y experiencia, tanto en médicos de primer contacto como en médicos especialistas, ya que la sospecha diagnóstica más una exploración física minuciosa son la piedra angular para un diagnóstico certero, lo que evita complicaciones postquirúrgicas y la alta mortalidad que puede desencadenar el retraso en el tratamiento.

\section{REFERENCIAS}

1. Cervantes CJ, Campos CS. Abdomen agudo. En: Gutiérrez SC, Arrumbarrena AV, Campos CS. Fisiología quirúrgica del aparato digestivo. $4 .^{\circ}$ ed. México: Manuel Moderno; 2012. pp. 512-528.

2. Macaluso CR, McNamara RM. Evaluation and management of acute abdominal pain in the emergency department. Int J Gen Med. 2012; 5: 789-797.

3. Bejarano M, Gallego CX, Gómez JR. Frecuencia de abdomen agudo quirúrgico en pacientes que consultan al servicio de urgencias. Rev Colomb Cir. 2011; 26: 33-41.

4. Montalva NS, Gurrea MR, Santis FC. Abdomen agudo de causa no especificada con un enfoque hacia la educación médica. Rev Medicina y Humanidades. 2012; 4: 14-23.

5. Casado-Méndez PR, Vallés-Gamboa ME, CorralesCaymari Y, Cabrera-Zambrano Y, Méndez-Jiménez O. Enfermedades trazadoras del abdomen agudo quirúrgico no traumático. AMC. 2014; 18: 269-282.

6. Bermúdez-Balado A, Rivero-Ramos A, BarroetabeñaRiol Y, Padierne-Olivera R. Caracterización de los pacientes con abdomen agudo quirúrgico atendidos en el Centro Médico de Diagnóstico Integral. Municipio Pampán. Mediciego [Internet]. 2012; 18. Disponible 
en: http://www.medigraphic.com/pdfs/mediciego/ mdc-2012/mdcs121i.pdf

7. Ronald AS, Russell GP. Abdomen agudo. En: Townsend CM, Beauchamp RD, Evers BM, Mattox KL. Sabiston Tratado de cirugía. 19. ${ }^{a}$ ed. España: Elsevier; 2013. pp. 1141-1159.

8. Montalvo-Javé EE, Rodea-Rosas H, Athié-Gutiérrez C, Zavala-Habib A. Abdomen agudo. Avances en su manejo y abordaje. Trauma. 2008; 11: 86-91.

9. Trentzsch H, Werner J, Jauch KW. Acute abdominal pain in the emergency department - a clinical algorithm for adult patients. Zentralbl Chir. 2011; 136: 118-128.

10. Brekke M, Eilertsen RK. Acute abdominal pain in general practice: tentative diagnoses and handling. A descriptive study. Scand J Prim Health Care. 2009; 27: 137-140.

11. Toorenvliet BR, Bakker RF, Flu HC, Merkus JW, Hamming JF, Breslau PJ. Standard outpatient reevaluation for patients not admitted to the hospital after emergency department evaluation for acute abdominal pain. World J Surg. 2010; 34: 480-486.

12. Pérez-Hernández JL, Teuffer-Carrion LT, Díaz-Aldana EV, Hernández-Guerrero S, Moron-Ventura P, NievesDuran $M$, et al. Dolor abdominal en pacientes geriátricos evaluados en el servicio de urgencias en un hospital de tercer nivel. Rev Gastroenterol Mex. 2010; 75: 261-266.

13. Berbel-Tornero $O$, Clemente-Yago F, García-Rodríguez C, Pereda-Pérez A. Dolor abdominal crónico y recurrente. Asociación Española de Pediatría. Libro electrónico 4: 29-35. Disponible en: https://www. aeped.es/sites/default/files/documentos/dar.pdf

14. de Quesada-Suárez L, Ival-Pelayo M, González-Meriño CL. La escala de Alvarado como recurso clínico para el diagnóstico de la apendicitis aguda. Rev Cubana Cir. 2015; 54: 121-128.

15. Khan I, ur Rehman A. Application of Alvarado scoring system in diagnosis of acute appendicitis. J Ayub Med Coll Abbottabad. 2005; 17: 41-44.

16. Moreno VJ, Marín LM, Rabanaque I. Dolor abdominal agudo. En: Blanco-Echeverría A, Cea-Calvo L, GarcíaGil ME, Menassa A, Moreno-Cuerda VJ, MuñozDelgado G, et al. Manual de diagnóstico y terapéutica médica. 5. ${ }^{\text {a }}$ ed. Madrid: MSD; 2003. pp. 67-76.
17. Glasgow RE, Mulvihill SJ. Dolor abdominal, incluido el abdominal agudo. En: Feldman M, Sleisenger $\mathrm{MH}$, Friedman L. Enfermedades gastrointestinales y hepáticas. 7.a ed. Madrid: Editorial Médica Panamericana; 2004. pp. 75-84.

18. Parrilla-Paricio P, García-Valdecasas Salgado JC, Targarona-Soler EM. Abdomen agudo. En: Farreras P, Rozman C, editores. Medicina interna. $15 .^{\mathrm{a}}$ ed. Madrid: Ed. Elsevier; 2004. pp. 168-171.

19. Ochando G, Millán MC, Pereda A. Dolor abdominal de origen psicosomático. An Peditr Contin. 2006; 4: 213-218.

20. Berbel O, Ortuño J, Pereda A. Dolor abdominal crónico y dolor abdominal recurrente. An Pediatr Contin. 2006; 4: 205-212.

21. American Academy of Pediatrics Subcommittee on Chronic Abdominal Pain. Chronic abdominal pain in children. Pediatrics. 2005; 115: 812-815.

22. Canavosso L, Carena P, Carbonell JM, Monjo L, Palas-Zúñiga C, Sánchez M, et al. Dolor en fosa iliaca derecha y score de Alvarado. Cir Esp. 2008; 83: 247251.

23. Balsano N, Cayten CG. Surgical emergencies of the abdomen. Emerg Med Clin North Am. 1990; 8: 399410.

24. García-Cabezudo J, Montoro MA, Canellas P, Pontaque M. Aproximación al paciente con dolor abdominal agudo (I y II). En: Montoro M, Bruguera M, Gomollón F, Santolaria S, Vilardell F. Principios básicos de

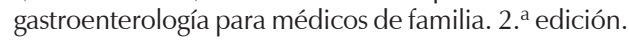
Madrid: Jarpyo Editores; 2002. pp. 751-782.

25. Jacobs DO. Apendicitis aguda y peritonitis. En: Kasper DL, Fauci AS, Hauser SL, Longo DL, Jameson JL, Loscalzo J. Harrison principios de medicina interna. 19. a edición. México: McGraw-Hill; 2016. pp. 19851988.

Correspondencia:

Dr. Salvador Eloy García-Valenzuela

Servicio de Cirugía General del Hospital Regional, ISSSTE.

Calzada Heroico Colegio Militar Núm. 875 Sur, Col. 5 de Mayo, 80000,

Culiacán, Sinaloa, México.

E-mail: dr.sgv_86@hotmail.com 